\title{
Analysis of Plane Waves Spectra in a Weakly-Disturbed Flat Reverberation Chamber by Information Theory Criteria
}

\author{
Kamel NAFKHA, Hedi RAGAD, Ali GHARSALLAH \\ CSEHF Research Unit, Faculty of Sciences of Tunis, Tunis El Manar University, Tunis, Tunisia \\ Kamel.nafkha@isim.rnu.tn, hedi.ragad@gmail.com, ali.gharsallah@gmail.com
}

Submitted November 20, 2017 / Accepted October 1, 2018

\begin{abstract}
This work aims to present the impact of disturbing the stationary modes in a rectangular-shaped reverberation chamber by small-dimensioned stirrers. The study focuses on plane wave's modeling of the electric field inside a flat cavity using information theory criteria. The emphasis is on anisotropy of the number of plane-waves distribution in the cavity and a strong increase near stirrers. The distributions of the plane wave spectra are studied for a set of reverberation chamber configurations at frequencies close to and far superior to the lowest usable frequency.
\end{abstract}

\section{Keywords}

Reverberation chamber, plane wave number, MDL criterion, AIC criterion, antenna array processing

\section{Introduction}

The reverberation chamber $(\mathrm{RC})$ is an essential tool to perform electromagnetic compatibility measurements, namely emissions, immunity, vulnerability, etc. It consists of an electromagnetic resonant cavity that permits a very large EM field with just modest-sized sources to which a device under test (DUT) is exposed. Variable boundary conditions such as vibrating walls, mechanical stirring, frequency sweep, are then used to generate a statistically homogeneous field (spatial and temporal homogeneity) around the DUT [1-3].

Several optimization studies have been carried out in order to have the most suitable configuration allowing for the most possible homogeneous field [2]. Other studies treated the chamber under another perspective; Hill [4] proposed a spatial isotropy hypothesis by resembling the EM field inside the chamber to a plane wave's superposition. This widened the applications of RC to other fields such as propagation channel modeling, multi-path environment modeling [5], [6] as well as measuring the shielding effectiveness of materials [7], etc.
In this paper, the number of plane waves illuminating an array of antennas inside the chamber is studied in order to get a local description of plane wave spectra. This paper is organized in the following manner. In Sec. 2, we describe the principle of the algorithm for estimating the number of plane waves. In Sec. 3, the techniques used to improve the estimation capacity of the algorithm are presented, by reducing the correlation between the wavefronts. Section 4 exposes the set of studied configurations and the simulations results using Ansoft HFSS software for modeling the weakly-perturbed flat cavity. Finally, Section 5 presents the results about the plane wave's spectra and the conclusions deduced.

\section{Estimation of the Plane Wave's Numbers}

As in previous works [4], [5], the electric fields were expanded into a set of plane waves travelling inside the cavity. This technique of estimating plane wave's spectra is another way for understanding RC fundamentals. To deal with this matter, we have referred to antenna processing techniques. In this way, many algorithms were developed with main investigation to estimate directions of arrivals (DoA). Some of them need to know, a priori, the number of plane waves to estimate like MUSIC [8] and ESPRIT [9] while giving high DoA resolution but others like Fourier discrete transformation, do not require a number of plane waves, at the cost of low DoA resolution. When plane wave number estimation is necessary, many authors refer to information theory criteria [10]. The key idea of our development is to obtain local descriptions of plane wave's spectra by estimating their numbers rather than a complete DoA processing. It consists of placing an array of antennas inside a resonant reverberation chamber to detect the values of the electric field. The detected values form an observation vector $\mathbf{X}$. The autocorrelation of this observation leads to the covariance matrix $\mathbf{R}_{\mathrm{xx}}$, used for estimation of plane wave's numbers by information theory criteria. 


\subsection{Signal Model}

Consider plane-wave fronts impinging on a uniform linear array of $M$ equidistant antennas, as shown in Fig. 1. Plane waves are coming from $P$ narrowband far-field sources and they are characterized by their complex magnitudes and their DoA $\theta_{i}, i \in\{1, \ldots, P\}$ defined from the normal axis of the antenna array.

The signal received from the source number (\#) $i$ and coming from the DoA $\theta_{i}$ on the antenna $\# m$ is written as follows:

$$
x_{m}^{i}(t)=S_{i}(t) \cdot \exp \left(-\mathrm{j} \frac{2 \pi \sin \left(\theta_{i}\right)}{\lambda} m d\right)+b_{m}(t)
$$

where $S_{i}(t)$ is the complex magnitude of the source $\# i$ and $b_{\mathrm{m}}(t)$ is an additive white noise on the antenna $\# m . \lambda$ is the wavelength of the respective plane wave and $d$ is the distance between two adjacent antennas.

Thus, the $M$ dimensional vector $\mathbf{X}^{i}(t)$ of the signals received on the antenna array at time $t$ from the source $\# i$ can be written as follows :

$$
\mathbf{X}^{i}(t)=\left[x_{0}^{i}(t) \quad x_{1}^{i}(t) \ldots x_{M-1}^{i}(t)\right] .
$$

$\mathbf{X}^{i}(t)$ is considered as a time observation on the antenna array at time $t$ from source $\# i$.

For a single source, it's clear that the signal $\mathbf{X}^{i}(t)$ has a harmonic waveform. For $P$ sources, $\mathbf{X}(t)$ is a linear combination of the $P$ plane wave contributions. The problem of detection is reduced to a harmonic signals' separation.

$$
\mathbf{X}(t)=\sum_{i=1}^{P} \mathbf{X}^{\mathrm{i}}(t)
$$

\subsection{Covariance Matrix}

Consider $\mathbf{X}\left(t_{n}\right)$ the time observation from the antenna array at time $t_{n}$. The covariance matrix $\mathbf{R}_{\mathrm{xx}}$ is the mean value of the time observations' autocorrelation such that:

$$
\mathbf{R}_{\mathrm{xx}}=\mathrm{E}\left(\mathbf{X}(t) \cdot \mathbf{X}(t)^{\mathrm{H}}\right)=\frac{1}{N} \sum_{t=t_{0}}^{t=t_{N-1}} \mathbf{X}(t) \cdot \mathbf{X}(t)^{\mathrm{H}}
$$

where $\mathrm{E}($.$) is the expectation operator, N$ is the number of time observations and $(.)^{\mathrm{H}}$ is the transpose-conjugate operator.

If all of the wave fronts are uncorrelated (i.e. incoherent sources), then the covariance matrix has a full rank equal to the number $P$ of independent wave fronts. The correlation between some wave fronts decreases the rank of the covariance matrix.

\subsection{Information Theory Criteria}

While the number of plane waves is known analytically in the canonical case of an empty parallelepiped cavity, it is no longer possible in the case of a cavity equipped by a stirrer. The key processing is then to detect the plane waves' number $P$, as defined in (3). The problem is thus reduced to model order selection. To do so, several information theory criteria based on maximum likelihood were developed. For example, Akaike [11] suggested the Akaike information criterion (AIC) for the estimation of the order $P$ of a parameterized statistical model, including the term $P \cdot(2 M-P)$ as a penalization of the likelihood function, as follows:

$$
\operatorname{AIC}(P)=-\mathrm{L}(P)+P \cdot(2 \mathrm{M}-\mathrm{P})
$$

where $\mathrm{L}(P)$ is the log-likelihood function parameterized by $P$.

The estimator of plane waves' number is the value $\hat{P}$ that minimizes this quantity, such that:

$$
\widehat{P}=\arg \left(\min _{P} \operatorname{AIC}(P)\right) .
$$

Rissanen [12] proposed an equivalent criterion by the coding theory which corresponds to the Minimum Description Length (MDL) criterion, such that:

$$
\operatorname{MDL}(P)=-\mathrm{L}(P)+1 / 2 P \cdot(2 M-P) \cdot \log (N)
$$

where $N$ denotes the time observation number. The MDL estimator $\hat{P}$ is then given by:

$$
\widehat{P}=\arg \left(\min _{P} \operatorname{MDL}(P)\right) .
$$
follows:

The log-likelihood function $\mathrm{L}(P)$ is expressed as

$$
\mathrm{L}(P)=N \cdot \log \left(\frac{\prod_{i=P+1}^{M} \lambda_{i}}{\left(\frac{1}{M-P} \cdot \sum_{i=P+1}^{M} \lambda_{i}\right)^{M-P}}\right)
$$

with $P$ varying from 1 to $M, M$ is the number of antennas per array; $N$ is the number of time observations and $\lambda_{i}$ are the estimated covariance matrix eigen-values.

\section{Plane Waves Decorrelation Proce- dure}

The covariance matrix as defined in (4) remains an approximation because the number of time observation $N$ is generally limited in real cases (infinite number in theoretical definition). Therefore, this lack in independent time observations' number can severely degrade the covariance matrix rank.

In the case of the reverberation chamber and due to standing waves phenomenon, time variations of electromagnetic fields are no longer dependent of space variations. Thus, this results in a single time observation $\mathbf{X}(t)$ for one resonant mode. Consequently, the phase differences between the incident plane waves are in fixed ratios and they are then interpreted as fully correlated. As a result, the covariance matrix is of unit-rank that is no longer equal to the number $P$ of the impinging plane waves. 


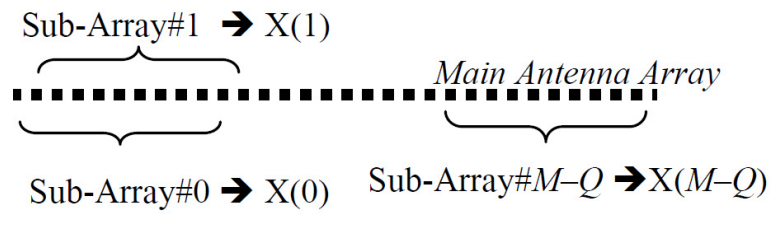

Fig. 1. Principle of spatial smoothing technique.

A decorrelation processing is implemented to overcome this problem and to ensure the non-singularity of the covariance matrix. The spatial smoothing technique [13] subdivides the main antenna array into $N$ overlapped antenna sub-arrays. Thus, this introduces space shifts between sub-arrays which in turn results in varying the phase conditions between relative sub-arrays' input observations.

Let $\mathbf{X}(n)$ be the time observation on \#n sub-array. Considering that each sub-array contains $Q$ equidistant antennas, we get a total number of $M-Q+1$ input observations. The spatial smoothing allows an increase in time observations at the cost of reduced antenna number per sub-array as shown in Fig. 1.

Therefore, the modified covariance matrix is nonsingular and is estimated as follows:

$$
\begin{aligned}
& \mathbf{R}_{\mathrm{xx}}= \\
& \mathrm{E}\left(\mathbf{X}(n) \cdot \mathbf{X}(n)^{\mathrm{H}}\right)=\frac{1}{M-Q+1} \cdot \sum_{n=0}^{n=M-Q} \mathrm{X}(n) \cdot \mathrm{X}(n)^{\mathrm{H}} .
\end{aligned}
$$

In some cases where plane waves' number is very important and DOA values are very close, spatial smoothing fails to restore the covariance matrix full rank.

As an alternative, we have used a second processing to enhance the accuracy of the covariance matrix. As the correlation matrix must have a Toeplitz form for completely uncorrelated signals, the $\mathbf{R}_{\mathrm{xx}}$-matrix diagonals must be constant. To reduce the $\mathbf{R}_{\mathrm{xx}}$ diagonals' coefficients variation [14], this processing consists of averaging the previously smoothed covariance matrix (forward) and the one obtained with the same antennas but ranged in a decreasing order (backward), i.e. from the last one ( $\# Q-1)$ to the first (\#0). Let $\mathbf{R}_{\mathrm{F}}$ be the forward covariance matrix and $\mathbf{R}_{\mathrm{B}}$ the backward one, the average covariance matrix $\mathbf{R}_{\mathrm{FB}}$ is given by:

$$
\mathbf{R}_{\mathrm{FB}}=\left(\mathbf{R}_{\mathrm{F}}+\mathbf{R}_{\mathrm{B}}\right) / 2 \text {. }
$$

This averaging process also contributes to increase of the number of antennas per sub-array.

\section{Flat Cavity Model}

The studied reverberation chamber is a flat-geometrical cavity with $a=3.105 \mathrm{~m}$ along $x$-axis, $b=2.475 \mathrm{~m}$ along $y$-axis and $c=0.01 \mathrm{~m}$ along $\mathrm{z}$-axis. For such configuration, as it can be seen, $c$ is neglected in comparison to $a$ and $b$, so the first resonant modes are transverse magnetic (TM). Indeed, the $z$-axis dimension is very small and the electromagnetic fields have two-dimensional spatial distri- butions. The simulations of a $2 \mathrm{D}$ RC are sufficient to obtain the resonant frequencies and electric field cartographies required for plane waves' spectra analysis.

The key idea behind mechanical stirred reverberation chamber studies is to disturb the EM field by introducing a metallic disturber that is well known as stirrer in order to get the statistical homogeneity of the EM field spatial distribution. The aim of our study is to disturb the EM field in a weak way by a stirrer with reduced dimensions in comparison to the RC dimensions.

The Ansoft HFSS software based on finite-element method is used to simulate the $2 \mathrm{D}$ reverberation chambers. The studied RC has Lowest Usable Frequency (LUF) of about $6 f_{0}$, with $f_{0}$ is the empty cavity cut-off frequency [15] (i.e. $465 \mathrm{MHz}$ ). The electric field cartography is then obtained at $10 \mathrm{~mm}$ equidistant points that can be considered as electric field antennas. Also, the chamber walls as well as the stirrer are taken to be perfectly conducting.

\section{Results}

Let consider a line of cells of the $E_{z}$-field cartography grid. This line of real values forms an observation vector of $M$ components, as shown in Fig. 3. The preprocessing spatial smoothing technique decomposes the main array into sub-arrays of $Q=M / 2$ antennas and the total number of observations is equal to $N=M / 2+1$.

To quantify the number of plane waves in different regions of the $\mathrm{RC}$, the study consists of moving the array of antennas parallel to the $y$-axis into $x$-directions starting from the origin in steps of $10 \mathrm{~mm}$, as described in Fig. 3. For each position of the array, the spatial smoothing technique has been used to subdivide this main array of 248 antennas into several overlapped sub-arrays of 124 antennas, thus allowing calculating the average covariance matrix using (10). The average number $\hat{P}$ of plane waves at each position of the antenna array is estimated using the two different information theory criteria according to (6) and (8). In this way, we get the local descriptions of the plane wave number according to $x$-direction scan.

The same scan is repeated with another main antenna array subdivided into sub-arrays but moved parallel to $x$ axis to get local plane wave spectra number according to $y$ direction. The low-left corner of the cavity is considered as the coordinates' origin.

To show the performance of our method, the case of an empty flat cavity is studied at the resonant frequency of $940.63 \mathrm{MHz}$ which corresponds to $\mathrm{TM}_{5150}$ mode. In this case, the electric field can be analytically expanded in 4 planes waves [5]. For reason of symmetry of plane waves' DoA to the antenna array axis, the estimated number will be half the real number. Just to show the accuracy of our method, we limit this study to only $x$-direction scan, and the estimated numbers, as shown in Fig. 2, agree well with theoretical numbers. 


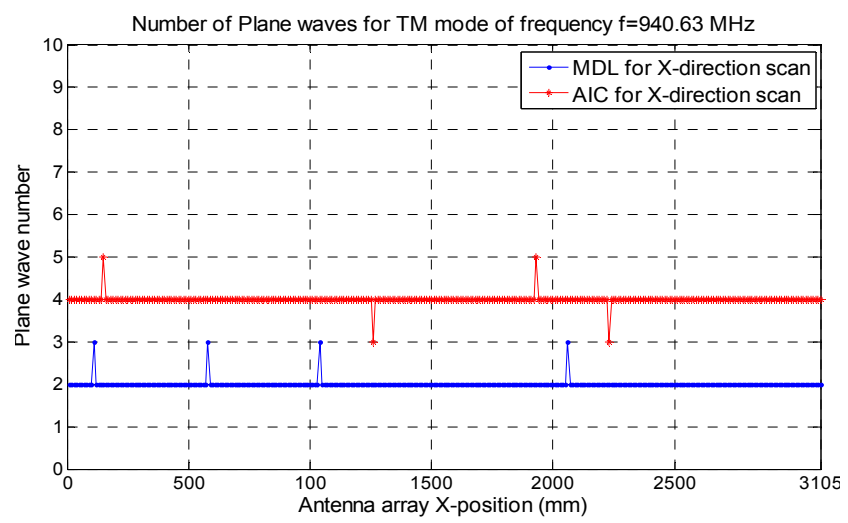

Fig. 2. Plane wave average numbers estimated when scanning $\mathrm{x}$-direction for an empty cavity case.

\subsection{Flat Cavity with One Stirrer}

In the first configuration case, a metallic disc-shaped stirrer of a radius $r=37.5 \mathrm{~mm}$ is introduced at the coordinates $x=450 \mathrm{~mm}$ and $y=450 \mathrm{~mm}$ from the coordinates origin. The stirrer dimensions remain very small in comparison to the RC geometry $(2 r=3 \%$ of $b$ dimension), as shown in Fig. 3 .

The first studied mode has a frequency equal to 938.15 MHz, which is considered as close to the LUF. Figure 3 shows the electric field cartography and the antenna arrays scanning the entire cavity in $x$ and $y$ directions.

Figure 4 shows the number of plane waves detected on each antenna array position when scanning the $x$-direction by AIC and MDL criteria. It is clear that the plane waves' number increases greatly in the vicinity of the disk due to the diffraction phenomena. This number decreases rapidly as we move far from the disk, which shows stability on the curve around a constant value. The same phenomena are observed near the disk edges when scanning the y-direction in the same way, as shown in Fig. 5.

We notify that for more clear figures, the number of plane waves' view is limited to the value of 80 .

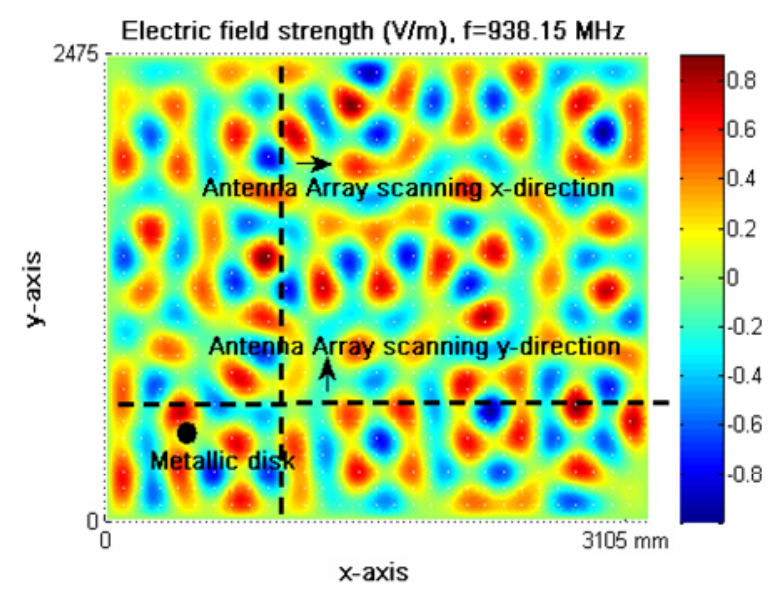

Fig. 3. Flat cavity equipped with one stirrer and the electric field strength cartography.

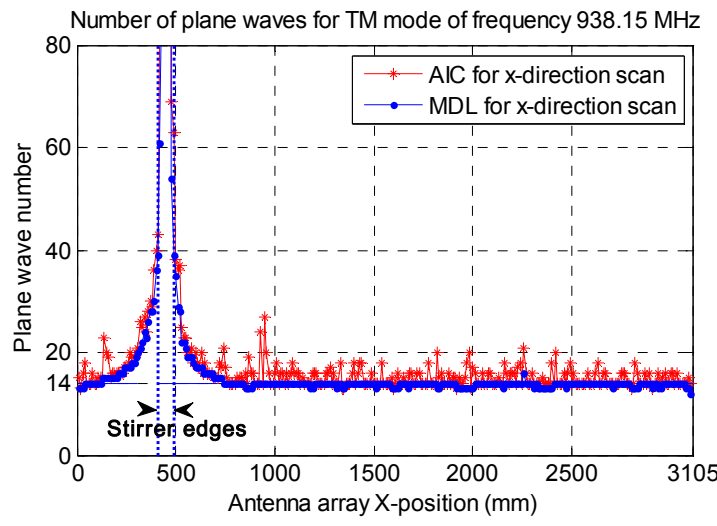

Fig. 4. Plane wave average numbers estimated when scanning $\mathrm{x}$-direction.

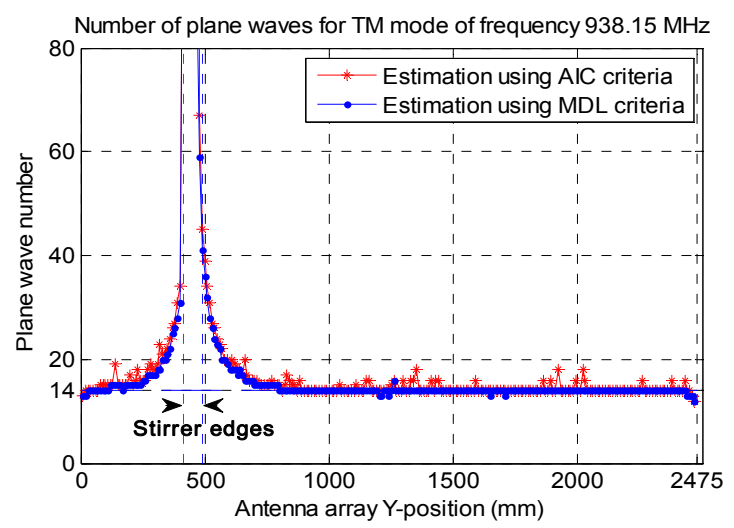

Fig. 5. Average plane wave number estimated when scanning y-direction.

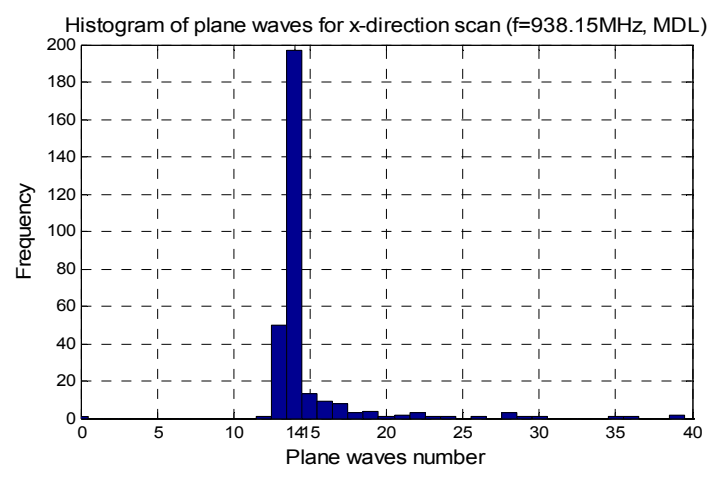

Fig. 6. Histogram of the plane waves numbers for $\mathrm{x}$-direction scan.

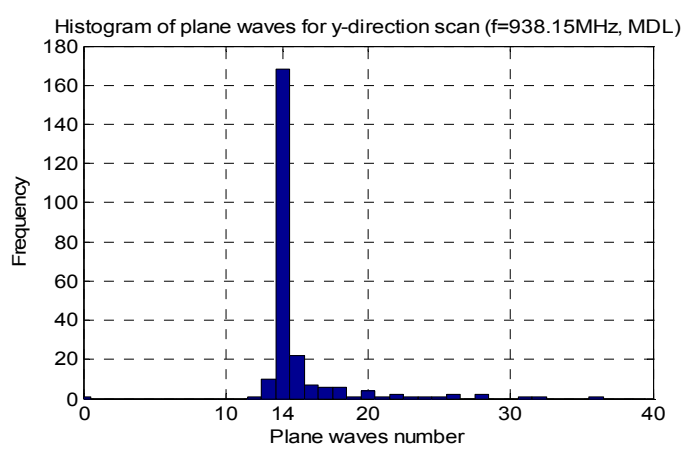

Fig. 7. Histogram of the plane waves numbers for $y$-direction scan. 
It is also clear that the MDL criterion estimates the plane waves' number in a better way than the AIC criterion, which reveals curves with less oscillation. We retain the MDL criterion for the following studies.

To precisely determine the constant value of the number of plane waves that are far from the stirrer forming the electric field, a histogram is used to classify the estimated numbers by frequency of occurrence in each of the positions of the scanning antenna array for $x$-direction and $y$-direction, as respectively shown in Fig. 6 and Fig. 7. The most probable obtained value either for scanning the $x$-direction or the $y$-direction is 14 .

Another resonant mode is studied with a frequency of $2.037 \mathrm{GHz}$ which is large superior to the LUF of the reverberation chamber. Figures 8 and 9 show the average estimated numbers of the plane wave illuminating the scanning antenna array for each $x$-position and $y$-position, respectively, while Figure 10 and Figure 11 show histograms of occurrences of frequencies of plane waves numbers for each scan direction. As shown in the previous paragraph we can observe the important diffraction phenomena near the disk edges.

Figure 12 exhibits a relative comparison between plane waves' numbers estimated by the MDL criterion for the two studied modes by scanning $x$-direction. It can be clearly deduced that the plane waves' spectrum becomes denser at high frequencies.

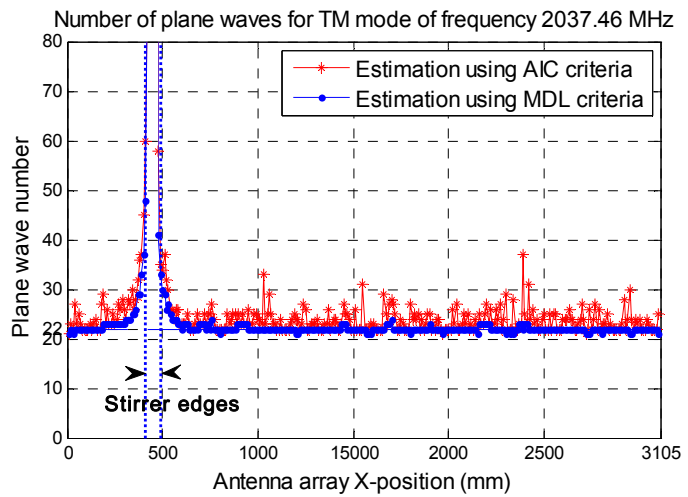

Fig. 8. Average plane wave number estimated when scanning $\mathrm{x}$-direction.

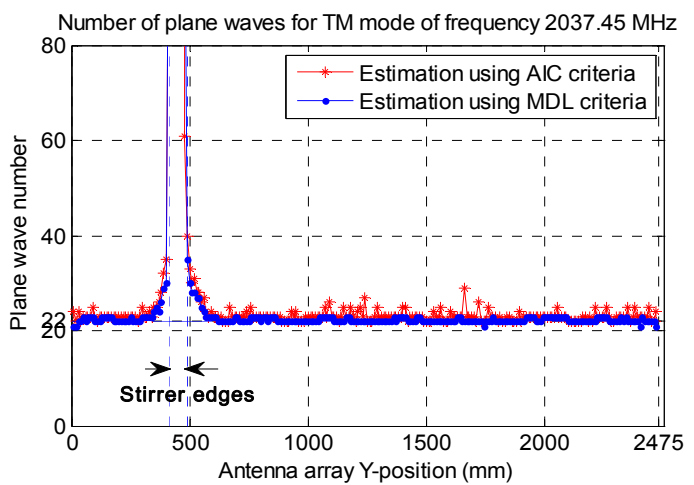

Fig. 9. Average plane wave number estimated when scanning $\mathrm{y}$-direction.

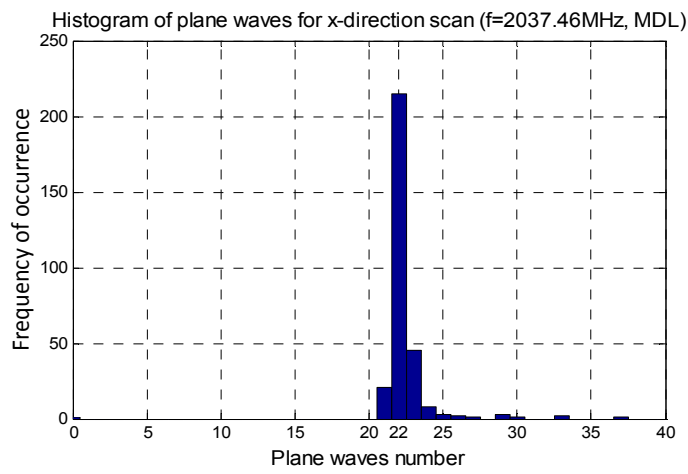

Fig. 10. Histogram of the plane waves numbers for $\mathrm{x}$-direction scan.

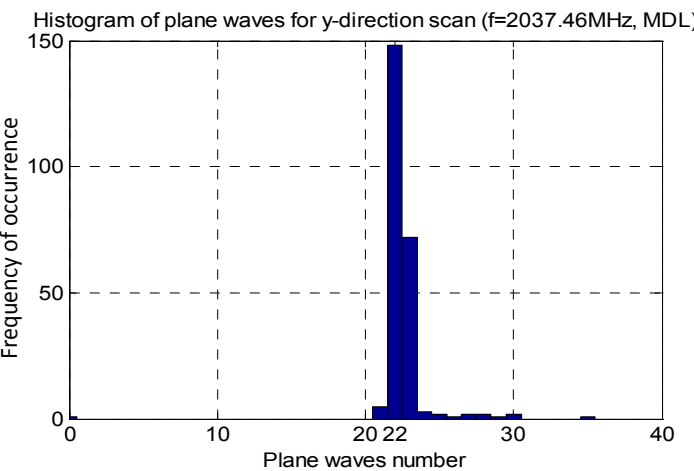

Fig. 11. Histogram of the plane waves numbers for $y$-direction scan.

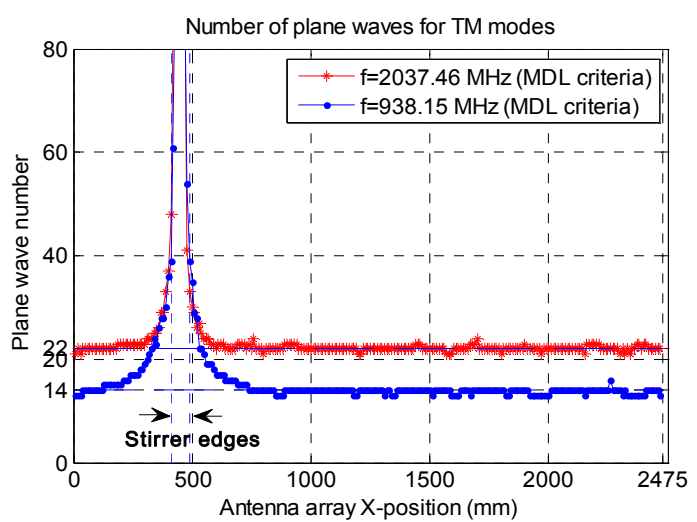

Fig. 12. Comparison between the MDL criteria results for two different modes (case of $x$-direction scan).

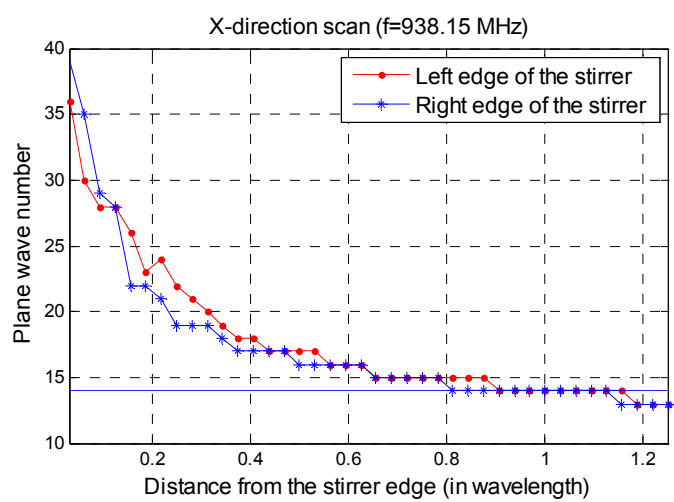

Fig. 13. Plane wave estimated numbers near the stirrer left and right edges. 


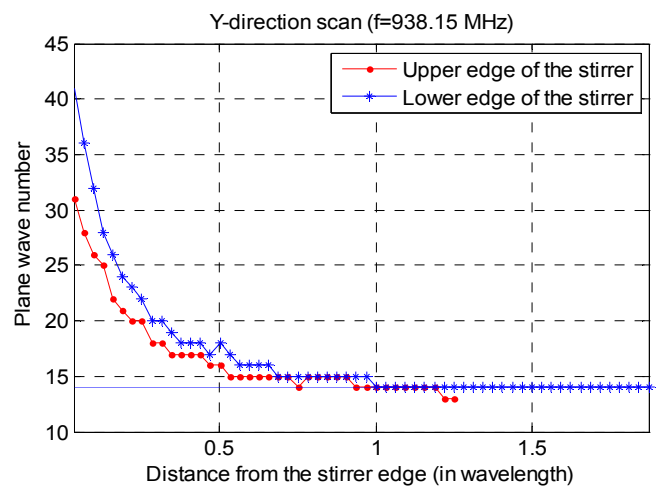

Fig. 14. Plane wave estimated numbers near the stirrer lower and upper edges.

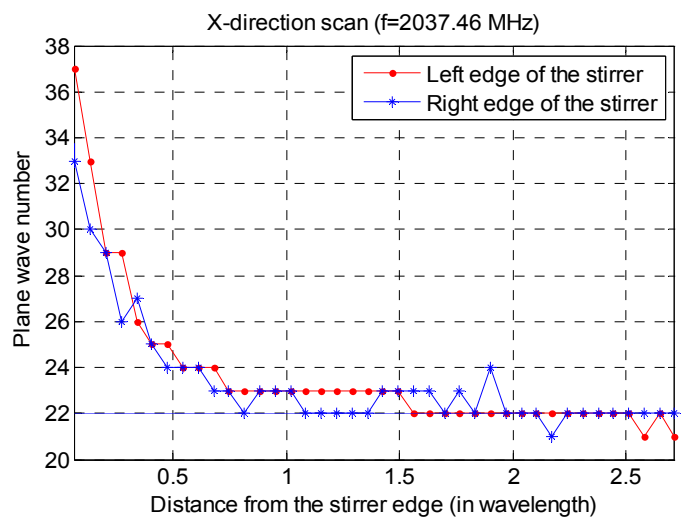

Fig. 15. Plane wave estimated numbers near the stirrer left and right edges.

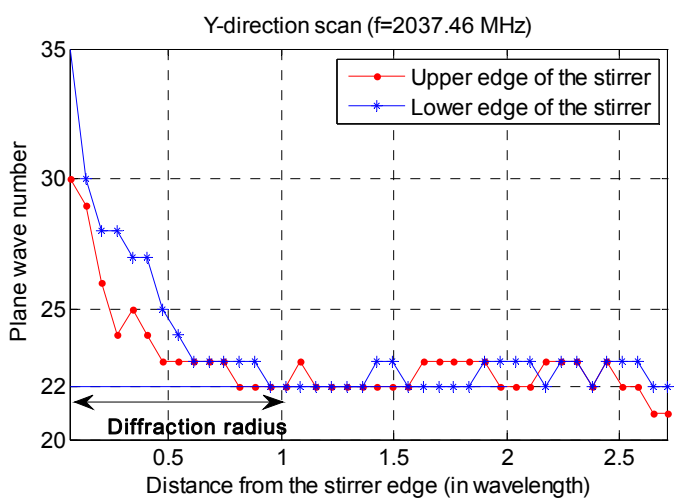

Fig. 16. Plane wave estimated numbers near the stirrer lower and upper edges.

\begin{tabular}{|c|c|c|c|}
\hline $\begin{array}{c}\text { Frequency } \\
{[\mathrm{MHz}]}\end{array}$ & Scan direction & $\begin{array}{c}\text { Average number of } \\
\text { plane waves }\end{array}$ & $\begin{array}{c}\text { Mean value of } \\
\text { diffraction } \\
\text { radius }\left(r_{\mathrm{d}}\right)[\lambda]\end{array}$ \\
\hline \multirow{2}{*}{938.15} & $\mathrm{x}$-scan (Fig.4) & 14 & 0.86 \\
\cline { 2 - 4 } & $\mathrm{y}$-scan (Fig.5) & 14 & 0.87 \\
\hline \multirow{2}{*}{2037.46} & $\mathrm{x}$-scan (Fig.8) & 22 & 1.18 \\
\cline { 2 - 4 } & $\mathrm{y}$-scan (Fig.9) & 22 & 0.88 \\
\hline
\end{tabular}

Tab. 1. Different estimated plane waves' numbers and diffraction radii.

The curves near the stirrer edges show a correlation to the mode frequency, i.e. the free space wavelength relative to the frequency of the mode. To quantify this correlation, we have first studied these curves near the two edges of the stirrer. The left edge is the one near the origin and the right edge is on the other side.

Figure 13 presents the curves of the plane waves' numbers that are near the stirrer edges with a distance normalized by the free space wavelength $(\lambda)$ relative to the mode frequency of $938.15 \mathrm{MHz}$. The left edge is near the $x$-axis origin and the right edge is on the other side of the axis.

Figure 14 shows the results of the same study but for scanning the $y$-direction where the lower edge is near the $y$ axis origin and the upper edge is on the other side of the axis.

It is clear that the curve of the plane wave number follows the same law for both $x$-direction and $y$-direction. This number requires an approximate distance of $0.8 \lambda$ to become constant, i.e equal to 14 .

The same study is repeated for the second mode with frequency of $2037.45 \mathrm{MHz}$. Figure 15 and Figure 16 exhibit the curves of the plane waves' estimated numbers around 4 edges of the stirrer.

Table 1 summarizes the previously studied cases and shows the constant value of the plane waves which form the $E z$-field cartography for each case. It also shows the distance from the edge of the stirrer that is required for the value to become constant. This distance is labeled the diffraction radius $\left(r_{\mathrm{d}}\right)$, as shown in Fig. 16.

We conclude that a distance of lambda is at least required to get a stable and less disturbed spectrum near the stirrer.

\subsection{Flat Cavity with Two Stirrers}

Here, a second metallic disk (stirrer \#2) with the same dimensions as stirrer\#1 is added to the cavity at the coordinates $x=2212.5 \mathrm{~mm}$ and $y=1837.5 \mathrm{~mm}$.

This new configuration is studied at the frequency $f$ of 2037.72 MHz (above the LUF). Figure 17 describes the electric field strength cartography for the studied TM-mode of frequency $f$.

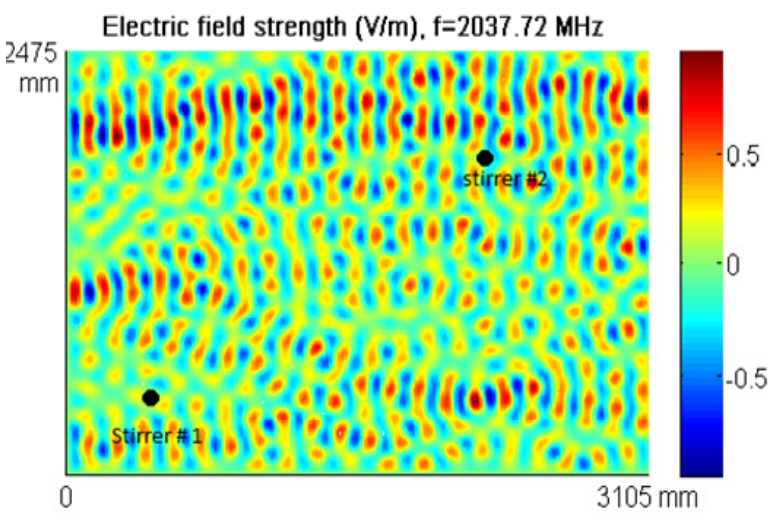

Fig. 17. Electric field cartography of TM mode of frequency $f=2037.72 \mathrm{MHz}$ in the RC equipped with two stirrers. 


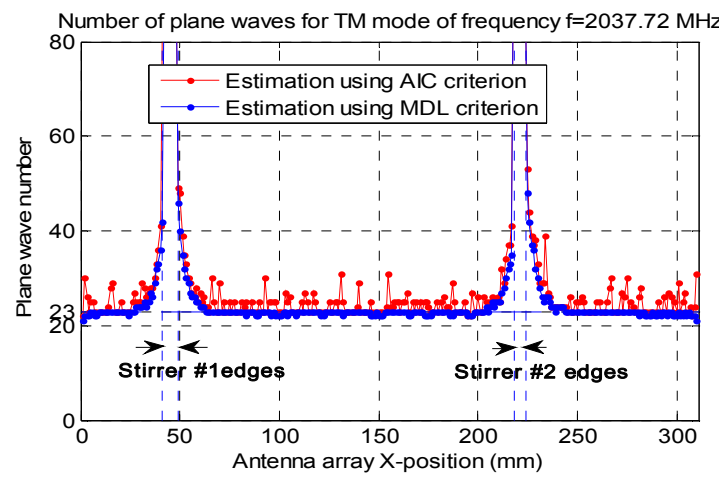

Fig. 18. Estimated numbers of plane waves for the resonant mode of $2037.72 \mathrm{MHz}$ in the RC equipped with two stirrers.

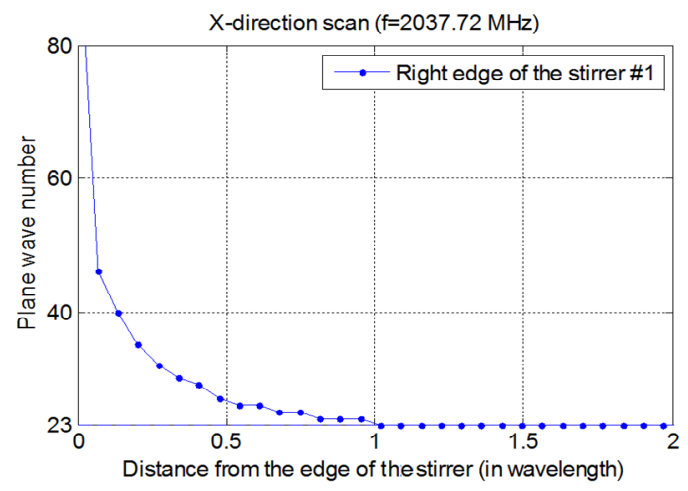

Fig. 19. Number of plane waves versus the normalized distance near the stirrer \#1.

Figure 18 shows the plane waves' numbers estimated when scanning $x$-direction. We observe that the estimated average number of plane waves is very large near the stirrers and it rapidly descends as soon as scanning antennas move away from them. Clearly, the plane wave spectrum becomes more stable beyond a distance of $\lambda=c / f$ (the diffraction zone) where $c$ is the speed of light in the vacuum and $f$ is mode frequency, as shown in Fig. 19.

By adding a measurement antenna into the cavity (stirrer \#2), we can qualify the amount of disturbance that can be introduced to the spatial distribution of the electric field.

The algorithm was proved in an empty cavity (theoretical case) and in simulated cases of the disturbed RC. The study shows the impact of a small-dimensioned stirrer on the field distribution and how much it can affect plane wave's spectra. The introduction of a measurement tool as small as possible will disturb the whole field distribution and correlate the spectra estimation. At this level of study, this algorithm is limited to simulated cases but de-noising techniques have to be used to eliminate plane wave's number introduced by the measurement process.

\section{Conclusion}

Good agreement of the results with the theoretical case of the empty cavity has been demonstrated by our algorithm. This leaded to a good description of the plane wave numbers by extrapolation to the case of the disturbed cavity. The main contribution was a precise description of the diffraction phenomenon, and an estimation of an accurate value of the diffraction zone radius around the stirrer. Our study has contributed to quantify the amount of disturbance added to EM fields when inserting a measurement tool, which is a hard task to do in reality due to its influence on the cavity behavior.

\section{References}

[1] HUANG, Y., EDWARDS, D. J. An investigation of electromagnetic fields inside a moving wall mode-stirred chamber. In the $8^{\text {th }}$ International Conference on Electromagnetic Compatibility. Edinburgh (UK), 1992, p. 115-119. ISBN: 0-85296-554-0

[2] CLEGG, J., MARVIN, A. C., DAWSON, J. F, et al. Optimization of stirrer designs in a reverberation chamber. IEEE Transactions on Electromagnetic Compatibility, November 2005, vol. 47, no. 4, p. 824-832. DOI: 10.1109/TEMC.2005.860561

[3] HILL, D. A. Electronic mode stirring for reverberating chambers. IEEE Transactions on Electromagnetic Compatibility, November 1994, vol. 36, no. 4, p. 294-299. DOI: 10.1109/15.328858

[4] HILL, D. A. Plane wave integral representation for fields in reverberation chambers. IEEE Transactions on Electromagnetic Compatibility, August 1998, vol. 40, no. 3, p. 209-217. DOI: $10.1109 / 15.709418$

[5] ROSENGREN, K., KILDAL, P. Study of distributions of modes and plane waves in reverberation chambers for the characterization of antennas in a multipath environment. Microwave and Optical Technology Letters, 2001, vol. 30, no. 6, p. 386-391. DOI: 10.1002/mop.1323

[6] KILDAL, P., CHEN, X., ORLENIUS, C., et al. Characterization of reverberation chambers for OTA measurements of wireless devices: physical formulations of channel matrix and new uncertainty formula. IEEE Transactions on Antennas and Propagation, 2012, vol. 60, no. 8, p. 3875-3891. DOI: 10.1109/TAP.2012.2201125

[7] HOLlOWAY, C. L., HILL, D. A., LADBURY, J., et al. Shielding effectiveness measurements of materials using nested reverberation chambers. IEEE Transactions on Electromagnetic Compatibility, May 2003, vol. 45, no. 2, p. 350-356. DOI: 10.1109/TEMC.2003.809117

[8] DEGROAT, R. D., DOWLING, E. M., LINEBARGER, D. A. The constrained MUSIC problem. IEEE Transactions on Signal Processing, March 1993, vol. 41, no. 3, p. 1445-1449. DOI: $10.1109 / 78.205753$

[9] ROY, R., KAILATH, T. ESPRIT - Estimation of signal parameters via rotational invariance techniques. IEEE Transactions on Acoustics Speech and Signal Processing, July 1989, vol. 37, no. 7, p. 984-995. DOI: $10.1109 / 29.32276$

[10] WAX, M., KAILATH, T. Detection of signals by information theoretic criteria. IEEE Transactions on Acoustics, Speech and Signal Processing, April 1985, vol. 33, no. 2, p. 387-392. DOI: 10.1109/TASSP.1985.1164557

[11] AKAIKE, H. Information theory and an extension of the maximum likelihood principle. In Proceedings of the 2nd International Symposium on Information Theory. Tsahkadsor (USSR), September 1971, p. 267-281.

[12] RISSANEN, J. Modeling by shortest data description length. 
Automatica, 1978, vol. 14, no. 5, p. 465-471. DOI: $10.1016 / 0005-$ 1098(78)90005-5

[13] EVANS, J. E., JOHNSON, J. R., SUN, D. F. High resolution angular spectrum estimation techniques for terrain scattering analysis and angle of arrival estimation. In Proceedings of the $1^{s i}$ Acoustics, Speech, and Signal Processing Workshop on Spectral Estimation. Hamilton (Canada), August 1981, p. 134-139.

[14] PILLAI, S. U., KWON, B. H. Forward/backward spatial smoothing techniques for coherent signal identification. IEEE Transactions on Acoustics, Speech, and Signal Processing, January 1989, vol. 37, no. 1, p. 8-15. DOI: 10.1109/29.17496

[15] INTERNATIONAL ELECTROTECHNICAL COMMISSION. Electromagnetic Compatibility (EMC) - Part 4-21: Testing and Measurement Techniques - Reverberation Chamber Test Methods (IEC 61000-4-21). 224 pages. [Online] Cited 2011-01-27. Available at : https://webstore.iec.ch/publication/4191

\section{About the Authors ...}

Kamel NAFKHA was born in Tunisia. He received his B.S. from the Higher School of Sciences and Technology of Tunis, Tunisia, in 2002 and his M.S. and Ph.D. degrees from Paris-Est University, France, in 2005 and 2009, respectively. He is an Associate Professor with the Univer- sity of Gabes, Tunisia. His research interests include reverberation chambers and antenna array processing.

Hedi RAGAD was born in Tunisia. He received his M.S. degree in Electronic Engineering from Marne La Vallée University France, in 2007, and the Ph.D. degree from University of Nantes, France. His research interests include dielectric resonator, antennas, and passive RF devices.

Ali GHARSALLAH received his B.S. degree in Radiofrequency Engineering from the Higher School of Telecommunication of Tunis, Tunisia, in 1986 and the Ph.D. degree from the Engineering School of Tunis, Tunisia, in 1994. Since 1991, he has been with the Department of Physics, Faculty of Sciences of Tunis, Tunisia. He is currently a Full Professor of Electrical Engineering and the General Director of Technological Studies in the Tunisian Ministry of Higher Education. His current research interests include smart antennas, array signal processing, multilayered structures, and microwave integrated circuits. He is the author of about 200 papers published in scientific journals and 300 conference papers. He supervised more than 40 Ph.D. and 100 Master students. Dr. Gharsallah was the chair of the IEEE MTT and AP-S Tunisian Chapters from 2008 to 2016 and since 2017 respectively. 